VOLUME 2 | NUMBER 2 | MARCH 2020

Available online at http://proceedings.worldconference.id.

ISSN: 2656-1174 (online)

\title{
Analysis of Strength, Stiffness, and Stability The Formwork Construction in LRT Jabodebek Project
}

\author{
Agyanata Tua Munthe and Muhammad Ardiansyah Noegroho \\ Faculty of Engineering, University Mercu Buana Jakarta, Indonesia \\ Agyanata.umb@gmail.com, ancha.noegroho@gmail.com
}

\begin{abstract}
Abstrak
The Upper Longitudinal Beam casting on the Jabodebek LRT Project requires a shoring to be able to withstand the workloads. To avoid the failure of formwork construction, formwork must meet the strength, stiffness, and stability requirements for each formwork component material. The analysis is carried out on the value of bending stress, deflection, and shear that occur in each component of formwork. From the analysis carried out each obtained as follows. Strength requirements with flexural stress values that occur in Plywood, Girder GT 24, and Steel Waller SRZ materials, each of which is smaller than $\sigma$ permit $=100 \mathrm{~kg} / \mathrm{cm} 2,70000 \mathrm{~kg} / \mathrm{cm} 2$, and $1200 \mathrm{~kg} / \mathrm{cm} 2$. Stiffness requirements with deflection values that occur on Plywood and Girder GT 24 material are $\Delta$ permit $=1 / 400$. Whereas the SRZ Steel Waller fulfills $\Delta$ permit $=1 / 240$. Stability requirements with shear stress values that occur in Plywood, Girder GT 24, and Steel Waller SRZ materials, each of which is smaller than $\tau$ permit $=12 \mathrm{~kg} / \mathrm{cm} 2$, $1400 \mathrm{~kg} / \mathrm{cm} 2$, and $696 \mathrm{~kg} / \mathrm{cm} 2$. Peri Up Shoring can support all formwork loads. So it can be said that the construction of the Upper Longitudinal Beam formwork is in a safe condition.
\end{abstract}

Keywords: Formwork, Bending Stress, Deflection, Shear Stress

\section{Introduction}

In the construction of concrete work, there area three main components that must be completed carefully and will succeed in the structural work. The three components are concrete mixture, concrete reinforcement and formwork.

Formwork is a temporary mold that is used to hold concrete as long as the concrete is poured and shaped in accordance with the desired shape. Because it functions as a temporary mold, formwork will be removed or dismantled if the concrete that has been poured has reached sufficient strength. To avoid formwork failures due to working loads and other factors, a formwork construction must meet the strength, stiffness and stability requirements. Formwork is said to be strong if when receiving loads - the workloads formwork material is not broken. The strength of formwork becomes a major component in producing quality structural dimensions that are in accordance with the plan. Formwork is said to be rigid, when when receiving loads - loads that work formwork material does not change shape. Formwork must also be stable, so that when receiving the formwork load does not collapse. This requirement must be met considering that formwork is work that is carried out repeatedly in high rise buildings and requires a large fee for rental costs and make it.

The construction of Light Rail Tansit Infrastructure Project is implemented by PT. Adhi Karya (Persero) Tbk. Formwork construction that is used for casting Transversal Beam and Lower Longitudinal Beam at Cawang Station is a steel profile structure fabricated by PT. Adhi Karya subcontractor's according to the needs of construction in the field. The construction process of the steel scaffolding construction requires lifting equipment such as a crawler / mobile crane. The steel formwork construction cannot be used for the construction of the Upper Longitudinal Beam because there is no wiggle room for the crane when assembling the steel scaffolding construction if the floor plate on the Concourse Level has been casted. Longitudinal Beam elevation is $\pm 4,588$ to the Concourse Level floor plate. So we need a formwork scaffolding system to withstand the forces that emerge when the Longitudinal Beam casting takes place. So this research aims to analyze the strength of the formwork structure against the flexural stress, deflection and shear stress of the material permits used so that it can be ensured that the formwork construction is safe. 


\section{Literatur Review}

According to Amien Sajekti (2009) quoted by Doloksaribu (2018), that formwork is a temporary mold for fresh concrete and as a support is used scaffolding (shore). [3]

According to Asiyanto (2010) quoted by Fitriansyah (2018), Formwork is a tool of concrete structures, for molding concrete into the desired shape, size, and controlling its position and alignment. [5]

Reference and scaffolding or formwork or formwork is a temporary construction in the form of a mall / mold on both the upper and lower sides of the desired concrete shape. Formwork functions as desired construction, while Scaffolding serves as a helper strengthening the form of construction.

According to Wigbout (1997), formwork construction works perform 5 functions, namely: [4]

1. Formwork determines the shape of the concrete construction to be made. The simple form of a concrete construction requires a simple formwork.

2. Formwork must be able to safely absorb the load caused by concrete species and various external loads and vibrations. In this case the change in shape that arises and the friction can be allowed as long as it does not exceed these tolerances.

3. Formwork must be able to be simply installed, removed and moved.

4. Preventing loss of wetness from new concrete.

5. Provides thermal insulation.

In planning a formwork, the following data are needed:
1. Concrete Self Weight
Reinforcement Concrete Self Weight
Wet concrete specific gravity
(q) = Volume $x$ Concrete Specific Gravity
$\mathrm{q}=2400 \mathrm{Kg} / \mathrm{m}^{3}=24 \mathrm{kN} / \mathrm{m}^{3}$
$\mathrm{q}=2500 \mathrm{Kg} / \mathrm{m}^{3}=25 \mathrm{kN} / \mathrm{m}^{3}$

2. Live Load

$\mathrm{q}=250 \mathrm{Kg} / \mathrm{m}^{2}=2500 \mathrm{~N} / \mathrm{m}^{2}$

Live load consist of the weight of workers, tools and belisting materials, vibrators, buckets, cast pipes and wheelbarrows.

3. Shock Load

$\mathrm{q}=100 \mathrm{Kg} / \mathrm{m}^{2}=1000 \mathrm{~N} / \mathrm{m}^{2}$

Shock load is the load that occurs due to concrete work that is jerking at the time of pouring and vibration by the vibrator during compaction.

Calculation Formula

According to F.Wigbout Ing. (1997), for each situation in casting, the flexure in a field can be calculated using the following formula: Simplified formula by oleh F.Wigbout (1997) In his book entitled "Bekisting (Kotak Cetak) Hal.133" published by Erlangga. [4]

Correction of M.Max, D.Max \& Deflection

$$
\begin{aligned}
& \sigma \text { Max }=\frac{M \cdot M a x}{W} \\
& \tau \text { Max }=\frac{D \cdot M a x \times S}{I \times b} \\
& \Delta \text { Max }=\frac{5 \times w \times l^{4}}{384 \times E \times I}
\end{aligned}
$$

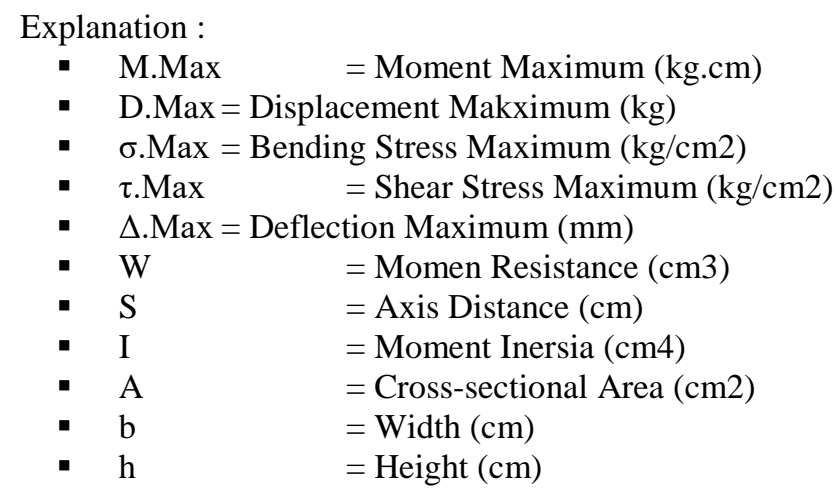


- W

- $\mathrm{E}$

- 1
$=$ Weight $(\mathrm{kg} / \mathrm{m})$

$=$ Modulus Elasticity $(\mathrm{kg} / \mathrm{cm} 2)$

$=$ Cross Section Length $(\mathrm{cm})$

\section{Research Methodology}

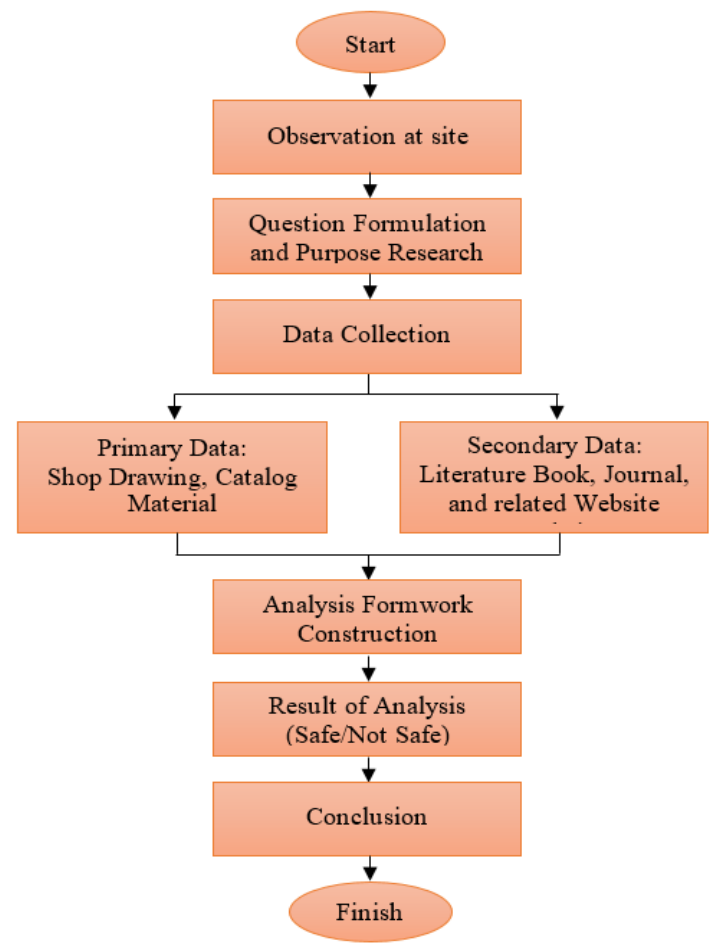

Figure 1. Flow Chart Research Method (Source: Author, 2020)

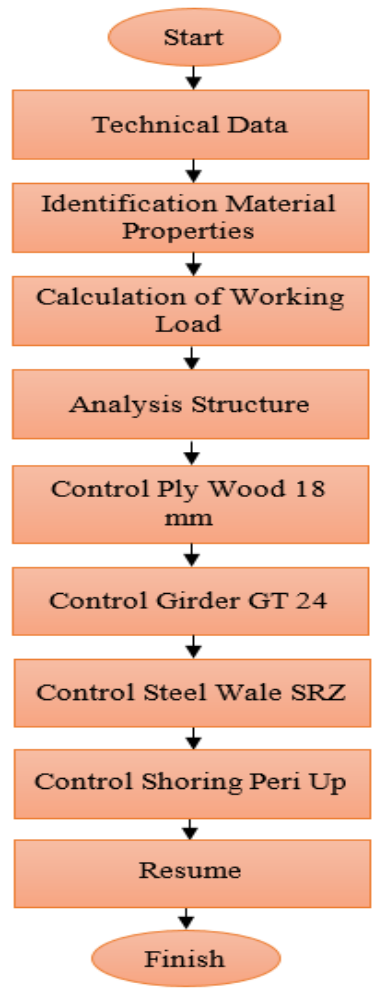

Figure 2. Flow Chart Analysis

(Source: Author, 2020)

Planning of a formwork shoring on the beam must of course take into account its strength, stiffness, and stability. In order to guarantee the achievement of strength, rigidity, and stability of formwork construction, it must be designed as a general structural design. Following is the flowchart methodology used in the completion of this Research.

\section{Results and Discussion}

\section{IV.1. Data Dimention Upper Longitudinal Beam}

Refer to Shop Drawing (LRT-CV-ST-DW-L2-TRLV-01 sd 47) getting form PT. Adhi Karya as a main contractor on Light Rail Transit JABODEBEK that is known the Upper Longitudinal Beam beam dimension data shown in Figure 3 and Table 1 below.

From the dimensional data in Figure 3 and Table 1, the calculation of shoring can be grouped because it has the same dimensions.

1. Block sizes 900x1000, namely: B1, B1R, B2, B3, B3L, B4, B4L, B5, B5L, B6, B6A, and B8L

2. Block Size 600 x1200, namely: B10

3. Block Size 800 x 1300, namely: B11 
VOLUME 2 | NUMBER 2 | MARCH 2020

Available online at http://proceedings.worldconference.id. ISSN: 2656-1174 (online)

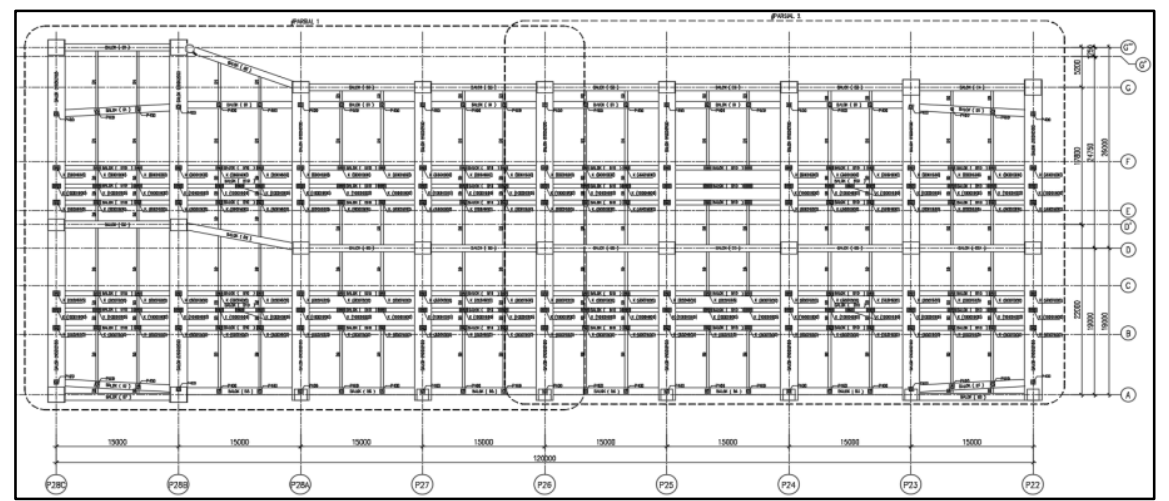

Figure 3. Site Plan Beam at Platform Level

(Source. : Data LRT Cawang BNN Station,2019)

Table 1. Dimention Upper Longitudinal Beam

\begin{tabular}{|l|l|l|l|l|l|c|}
\hline $\begin{array}{c}\text { Name of } \\
\text { Beam }\end{array}$ & $\begin{array}{c}\text { Dimention } \\
(\mathrm{mm})\end{array}$ & $\begin{array}{c}\text { Length } \\
(\mathrm{mm})\end{array}$ & $\begin{array}{c}\text { Name of } \\
\text { Beam }\end{array}$ & $\begin{array}{c}\text { Dimention } \\
(\mathrm{mm})\end{array}$ & $\begin{array}{c}\text { Length } \\
(\mathrm{mm})\end{array}$ \\
\hline B1 & $900 \times 1000$ & 12850 & & B5L & $900 \times 1000$ & 12800 \\
\hline B1R & $900 \times 1000$ & 12850 & & B6 & $900 \times 1000$ & 12850 \\
\hline B2 & $900 \times 1000$ & 13856 & & B6A & $900 \times 1000$ & 12850 \\
\hline B3 & $900 \times 1000$ & 12850 & & B8L & $900 \times 1000$ & 12850 \\
\hline B3L & $900 \times 1000$ & 12850 & & B10 & $600 \times 1200$ & 12850 \\
\hline B4 & $900 \times 1000$ & 12850 & & B11 & $800 \times 1300$ & 12850 \\
\hline B4L & $900 \times 1000$ & 12850 & & & & \\
\hline B5 & $900 \times 1000$ & 12850 & & & & \\
\hline
\end{tabular}

(Sorce : Author, 2020)

IV.2 Shoring Calculation for Beam 900*1000 mm

IV.2.1 Section Drawing

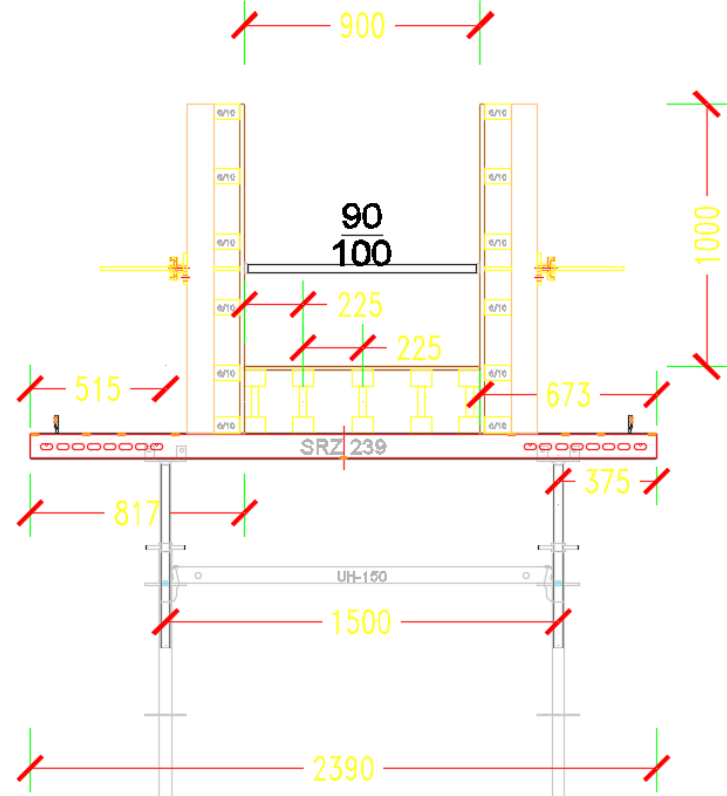

Figure 4. Upper Longitudinal Beam $900 \times 1000 \mathrm{~mm}$

(Source : Author, 2020) 
IV.2.2 Structural Analysis

IV.2.2.1 Control Ply Wood $18 \mathrm{~mm}$

Load to be used :

Concrete Load $=2500 \mathrm{~kg} / \mathrm{m}^{2} \times 1 \mathrm{~m} \times 0,9 \mathrm{~m}$

Live Load $=250 \mathrm{~kg} / \mathrm{m}^{2} \times 1 \mathrm{~m}$

Shock Load $=100 \mathrm{~kg} / \mathrm{m} 2 \times 1$

Selfweight of Plywood $=600 \mathrm{~kg} / \mathrm{m}^{3} \times 0,018 \times 1 \mathrm{~m}$

Total

$=2250 \mathrm{~kg} / \mathrm{m}$

$=100 \mathrm{~kg} / \mathrm{m}$

$=10,8 \mathrm{~kg} / \mathrm{m}$

Modeling Load
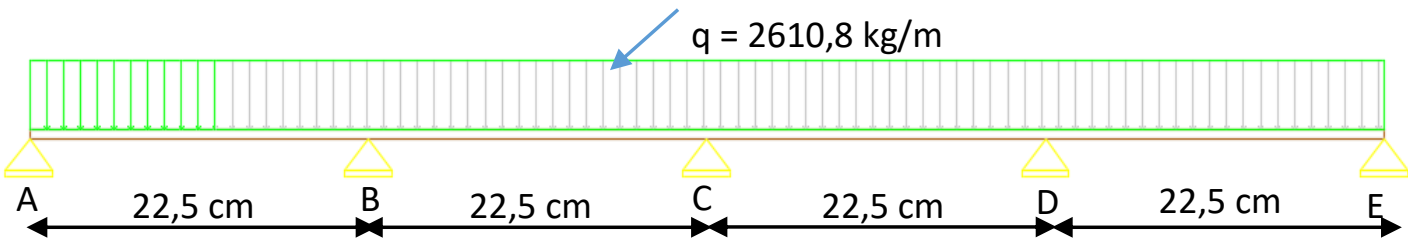

M.Max

According to F.Wigbout Ing. (1997), select configuration structure accordingly.

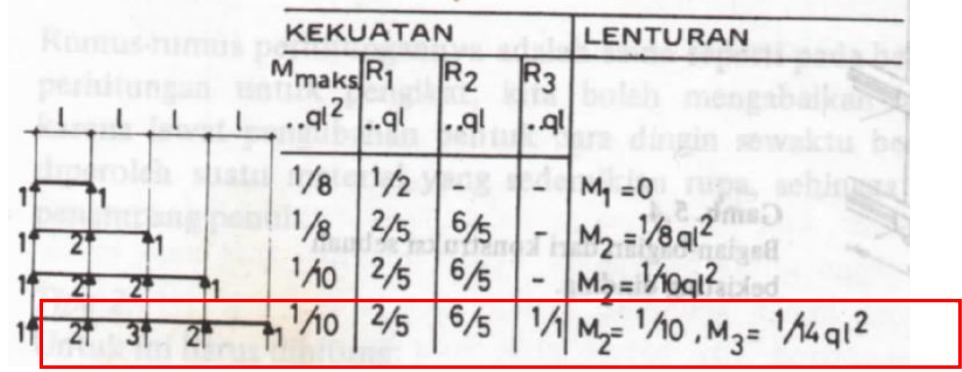

The moment maximum occurs in joint area no. 2 (B and $\mathrm{C}$ )

$M \operatorname{Max}=1 / 10 \times \mathrm{q} \times \mathrm{l}^{2}$

$$
\begin{aligned}
& =1 / 10 \times 2610,8 \times 0,225^{2} \\
& =12,711 \mathrm{~kg} \cdot \mathrm{m} \sim 1271,1 \mathrm{~kg} \cdot \mathrm{cm}
\end{aligned}
$$

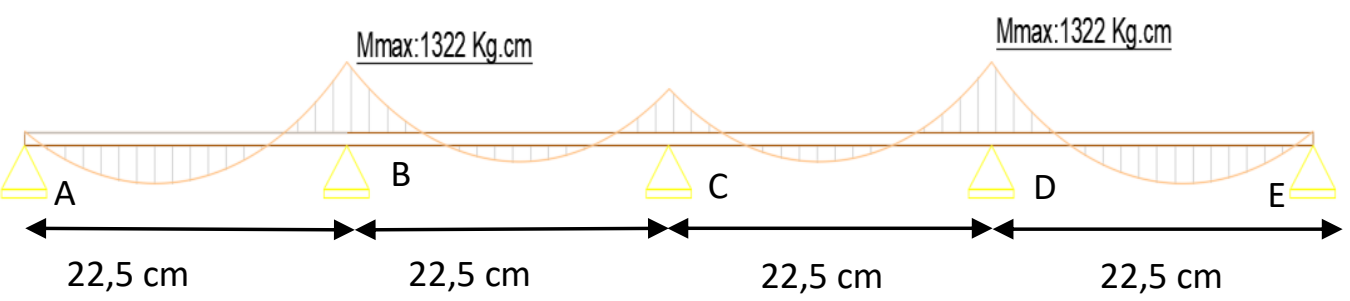

D.Max

$\mathrm{D}$ Max occurs in joint $\mathrm{B}$ and $\mathrm{D}$ :

$\mathrm{D} \operatorname{Max}=6 / 5 \times \mathrm{q} \times 1$

$=6 / 5 \times 2610,8 \times 0,225$

$=704,916 \mathrm{~kg}$ 


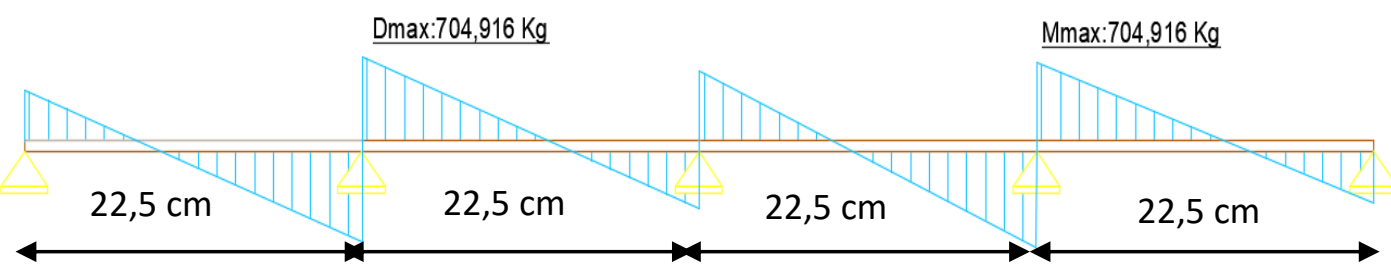

Correction of M.Max, D.Max \& Deflection

Properties Plywood $18 \mathrm{~mm}$

18

$\begin{array}{llcl}\mathrm{b} & =100 & \mathrm{~cm} \\ \mathrm{Ax} & =180 & \mathrm{~cm}^{2} \\ \mathrm{Ix} & =48.6 & \mathrm{~cm}^{4} \\ \mathrm{Wx}_{\mathrm{x}} & =54 & \mathrm{~cm}^{3} \\ \mathrm{Sx} & =40.5 & \mathrm{~cm}^{3} \\ \Delta \max & =4 / 400 & \\ \mathrm{E} & =100,000 \mathrm{~kg} / \mathrm{cm}^{2} \\ \sigma \text { ijin } & =100 \mathrm{~kg} / \mathrm{cm}^{2} \\ \tau \text { ijin } & =12 & \mathrm{~kg} / \mathrm{cm}^{2}\end{array}$

1) $\sigma$ Max $=\frac{M \cdot M a x}{W}=\frac{1271}{54}=23,537 \mathrm{~kg} / \mathrm{cm}^{2}$

$: 23,537 \mathrm{~kg} / \mathrm{cm}^{2}<\sigma_{\mathrm{ijin}}=100 \mathrm{~kg} / \mathrm{cm}^{2}$

2) $\tau$ Max $=\frac{D \cdot M a x \times S}{I \times b}=\frac{704,916 \times 40,5}{48,6 \times 100}=5,874 \mathrm{~kg} / \mathrm{cm}^{2}$

: $5,874 \mathrm{~kg} / \mathrm{cm}^{2}<\tau_{\mathrm{ijin}} 12 \mathrm{~kg} / \mathrm{cm}^{2}$

3) $\Delta$ Max $=\frac{5 \times \mathrm{wx} l^{4}}{384 \times \mathrm{EXI}}=\frac{5 \times 26,022 \times 22,5^{4}}{384 \times 100000 \times 48,6}=0,018 \mathrm{~cm}$

$: 0,018 \mathrm{~cm}<\Delta_{i j i n}=1 / 400=0,0562 \mathrm{~cm}$

\section{IV.2.2.2 Control Girder GT 24}

Load to be used :

Concrete Load $=2500 \mathrm{~kg} / \mathrm{m}^{2} \times 1 \mathrm{~m} \times 0,9 \mathrm{~m}$

Live Load $=250 \mathrm{~kg} / \mathrm{m}^{2} \times 1 \mathrm{~m}$

Shock Load $=100 \mathrm{~kg} / \mathrm{m} 2 \times 1$

Selfweight of Plywood $=600 \mathrm{~kg} / \mathrm{m}^{3} \times 0,018 \times 1 \mathrm{~m}$

Bekisting Samping $70 \mathrm{~kg} / \mathrm{m} 2$

Selfweight of Girder GT 24

$$
\begin{aligned}
& =2250 \mathrm{~kg} / \mathrm{m} \\
& =250 \mathrm{~kg} / \mathrm{m} \\
& =100 \mathrm{~kg} / \mathrm{m} \\
& =10,8 \mathrm{~kg} / \mathrm{m} \\
& =70 \mathrm{~kg} / \mathrm{m} \\
& =6 \mathrm{~kg} / \mathrm{m} \\
& =2686,8 \mathrm{~kg} / \mathrm{m}
\end{aligned}
$$

\section{Modeling Load}

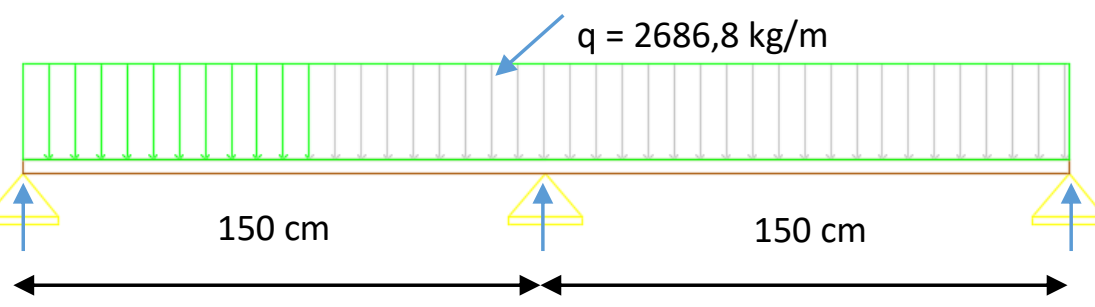




\section{M.Max}

According to F.Wigbout Ing. (1997), select configuration structure accordingly.

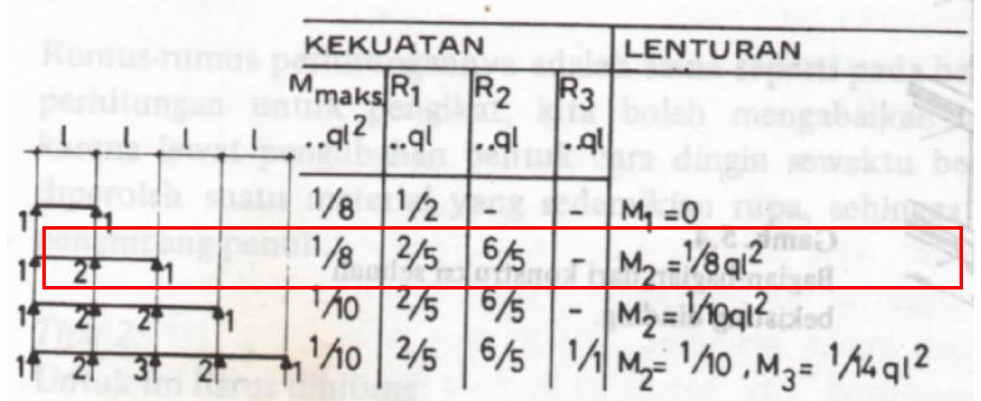

The moment maximum occurs in joint area no. 2 (Middle Joint)

$\operatorname{MMax}=1 / 8 \times \mathrm{q} \mathrm{x} \mathrm{l}^{2}$

$=1 / 8 \times 2686,8 \times 1,5^{2}$

$=755,662 \mathrm{~kg} \cdot \mathrm{m} \sim 75566,25 \mathrm{~kg} . \mathrm{cm}$

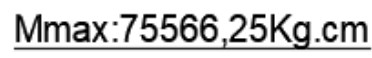

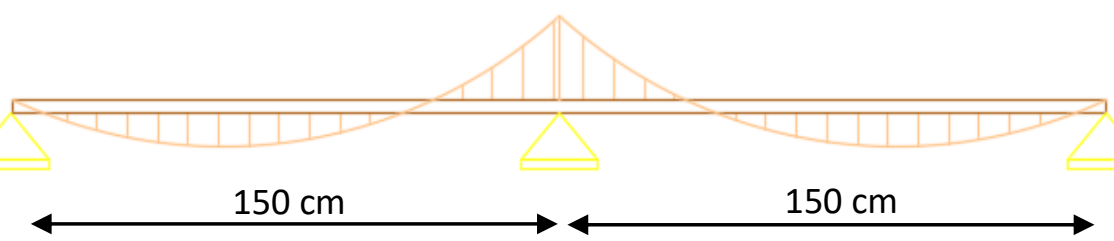

D.Max

D Max $=6 / 5 \times \mathrm{q} \times 1$

$=6 / 5 \times 2686,8 \times 1,5$

$=4836,24 \mathrm{~kg}$

\section{Dmax:4836,24 Kg}

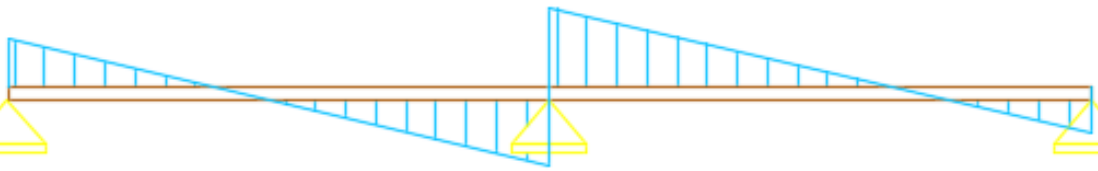

Correction of M.Max, D.Max \& Deflection

Properties Girder GT 24

$\begin{array}{llll}\mathrm{b} & =8 & \mathrm{~cm} \\ \mathrm{Ax} & =96 \mathrm{~cm}^{2} \\ \mathrm{Ix} & =8000 \mathrm{~cm}^{4} \\ \mathrm{Wx} & =700 \mathrm{~cm}^{3} \\ \mathrm{Sx} & =525 \mathrm{~cm}^{3} \\ \Delta \max & =\mathrm{L} / 400 \\ \mathrm{E} & =100,000 \mathrm{~kg} / \mathrm{cm}^{2} \\ \mathrm{M}_{\max } & =70000 \mathrm{~kg} \cdot \mathrm{cm} \\ D_{\max } & =1400 \mathrm{~kg}\end{array}$


1) $\sigma$ Max $=\frac{\text { M.Max }}{W}=\frac{75566,25}{700}=107,951 \mathrm{~kg} / \mathrm{cm}^{2}$

$: 107,951 \mathrm{~kg} / \mathrm{cm}^{2}<\sigma_{\text {ijin }} 70000 \mathrm{~kg} / \mathrm{cm}^{2}$

2) $\tau$ Max $=\frac{D \cdot \operatorname{Max} \times S}{I \times b}=\frac{4836,24 \times 525}{8000 \times 8}=39,672 \mathrm{~kg} / \mathrm{cm}^{2}$ $: 39,672 \mathrm{~kg} / \mathrm{cm}^{2}<\tau_{\mathrm{ijin}} 1400 \mathrm{~kg} / \mathrm{cm}^{2}$

3) $\Delta$ Max $=\frac{5 \times \mathrm{xw} l^{4}}{384 \times \mathrm{ExI}}=\frac{5 \times 26,082 \times 1,5^{4}}{384 \times 100000 \times 8000}=0,0001 \mathrm{~cm}$

$$
\text { : } 0,0001 \mathrm{~cm}<\Delta_{\mathrm{ijin}}=1 / 400=0,375 \mathrm{~cm}
$$

\section{IV.2.2.3 Control Steel Wale SRZ}

Load to be used :

Concrete Load $=2500 \mathrm{~kg} / \mathrm{m}^{2}$ x $1 \mathrm{~m}$ x $0,9 \mathrm{~m}$

Live $\mathrm{Load}=250 \mathrm{~kg} / \mathrm{m}^{2} \times 1 \mathrm{~m}$

Shock Load $=100 \mathrm{~kg} / \mathrm{m} 2 \times 1$

Selfweight of Plywood $=600 \mathrm{~kg} / \mathrm{m}^{3}$ x $0,018 \times 1 \mathrm{~m}$

Side Formwork 70 kg/m2

Selfweight of Girder GT 24

Selfweight of Steel Wale

Total

$$
\begin{gathered}
=2250 \mathrm{~kg} / \mathrm{m} \\
=250 \mathrm{~kg} / \mathrm{m} \\
=100 \mathrm{~kg} / \mathrm{m} \\
=10,8 \mathrm{~kg} / \mathrm{m} \\
=70 \mathrm{~kg} / \mathrm{m} \\
=6 \mathrm{~kg} / \mathrm{m} \\
=19,08 \mathrm{~kg} / \mathrm{m} \\
=2705,88 \mathrm{~kg} / \mathrm{m}
\end{gathered}
$$

\section{$\underline{\text { Modelling Load }}$}

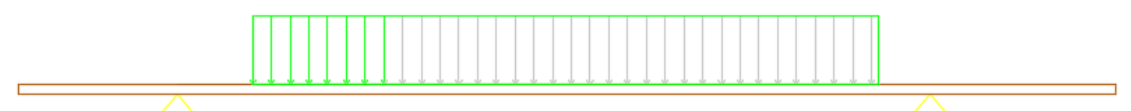

\section{$150 \mathrm{~cm}$}

$\underline{\text { M.Max }}$
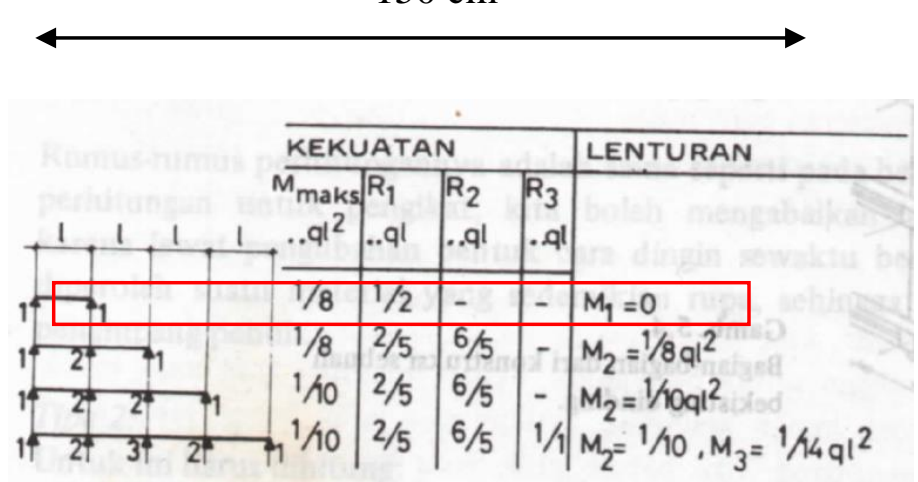

The moment maximum occurs in $1 / 8 \times \mathrm{q} \mathrm{x}^{2}$

$\operatorname{Max}=1 / 8 \times \mathrm{q} \mathrm{X}^{2}$

$=1 / 8 \times 2705,88 \times 1,5^{2}$

$=761,029 \mathrm{~kg} \cdot \mathrm{m} \sim 76102,88 \mathrm{~kg} \cdot \mathrm{cm}$

$\underline{\text { D.Max }}$

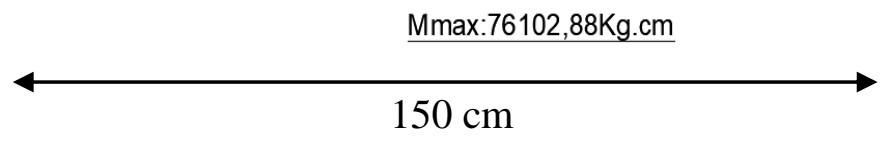

$\overline{\mathrm{D} \operatorname{Max}}=1 / 2 \times \mathrm{q} \times 1$

$$
\begin{aligned}
& =1 / 2 \times 2705,88 \times 1,5 \\
& =2029,41 \mathrm{~kg}
\end{aligned}
$$




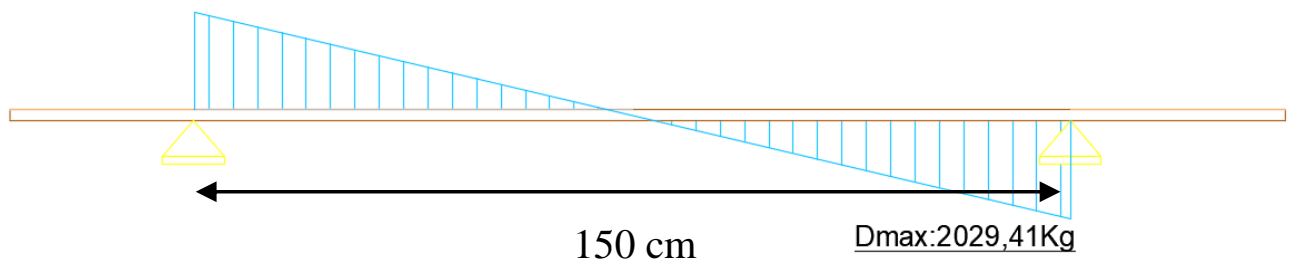

Correction of M.Max, D.Max \& Deflection

Properties Steel Waller

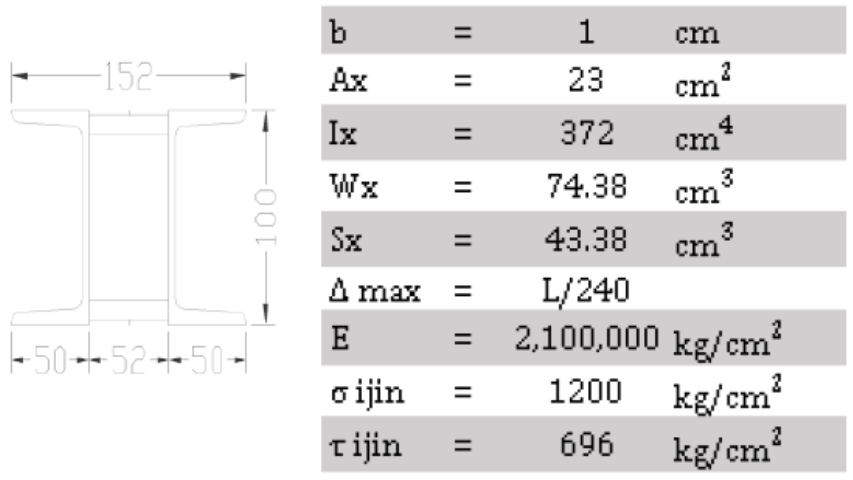

1) $\sigma$ Max $=\frac{M \cdot M a x}{W}=\frac{76102,88 \prime}{74,38}=1023,163 \mathrm{~kg} / \mathrm{cm}^{2}$

2) $\tau$ Max $=\frac{\text { D.Max } \times S}{I \times b}=\frac{\begin{array}{c}: 1023,163 \mathrm{~kg} / \mathrm{cm}^{2}<\sigma_{\mathrm{ijin}} 1200 \\ 2029,41 \times 43,38\end{array}=236,607 \mathrm{~kg} / \mathrm{cm}^{2}}{372 \times 1}=236,607 \mathrm{~kg} / \mathrm{cm}^{2}<\tau$.

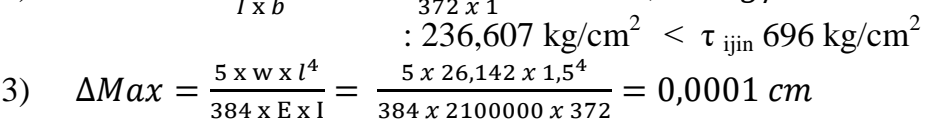

$$
\text { : } 0,0001 \mathrm{~cm}<\Delta_{\mathrm{ijin}}=1 / 240=0,625 \mathrm{~cm}
$$

\section{IV.2.2.4 Control Shoring}

Load to be used :

Concrete Load $=2500 \mathrm{~kg} / \mathrm{m}^{2} \times 1 \mathrm{~m} \times 0,9 \mathrm{~m}$

Live Load $=250 \mathrm{~kg} / \mathrm{m}^{2} \times 1 \mathrm{~m}$

Shock Load $=100 \mathrm{~kg} / \mathrm{m} 2 \times 1$

Selfweight of Plywood $=600 \mathrm{~kg} / \mathrm{m}^{3} \times 0,018 \times 1 \mathrm{~m}$

Side Formwork $70 \mathrm{~kg} / \mathrm{m} 2$

Selfweight of Girder GT 24

Selfweight of Steel Wale

Total

$$
\begin{gathered}
=2250 \mathrm{~kg} / \mathrm{m} \\
=250 \mathrm{~kg} / \mathrm{m} \\
=100 \mathrm{~kg} / \mathrm{m} \\
=10,8 \mathrm{~kg} / \mathrm{m} \\
=70 \mathrm{~kg} / \mathrm{m} \\
=6 \mathrm{~kg} / \mathrm{m} \\
=19,08 \mathrm{~kg} / \mathrm{m} \\
=2705,88 \mathrm{~kg} / \mathrm{m}
\end{gathered}
$$




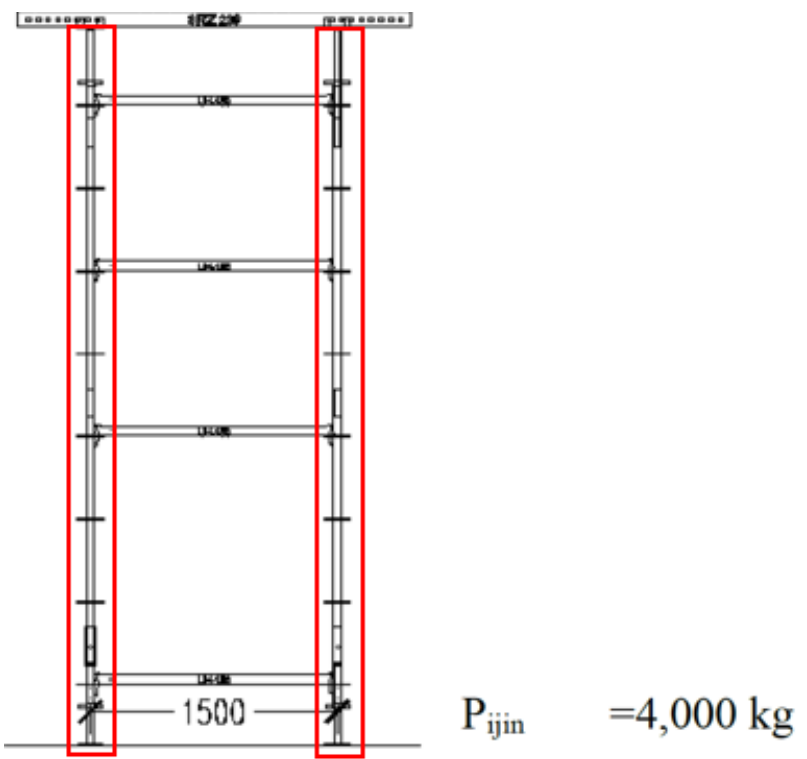

Calculation formula for 1 span :

$\mathrm{D} \operatorname{Max}=1 / 2 \times \mathrm{q} \times 1$

$=1 / 2 \times 2705,88 \times 1,5$

$=2029,41 \mathrm{~kg}<\mathrm{P}_{\mathrm{ijin}}=4000 \mathrm{~kg}$

\section{IV.2.3 Resume Control Stress, Strain, and Deflection}

The static calculation of the formwork shoring structure above, it can be concluded in Table 2 :

Table 2. Resume Control Stress, Strain, and Deflection Shoring for Beam $90 * 100 \mathrm{~cm}$

\begin{tabular}{|c|c|c|c|c|c|c|c|}
\hline Item & \multicolumn{5}{|c|}{ Control Value } & Unit & Status \\
\hline \multirow{3}{*}{ Plywood $18 \mathrm{~mm}$} & $\sigma_{\text {maks }}=$ & 23,537 & $<$ & $\sigma_{i j i n}=$ & 100 & $\mathrm{~kg} / \mathrm{cm}^{2}$ & $\mathrm{OK}$ \\
\hline & $\tau_{\text {maks }}=$ & 5,874 & $<$ & $\tau_{\mathrm{ijin}}=$ & 12 & $\mathrm{~kg} / \mathrm{cm}^{2}$ & $\mathrm{OK}$ \\
\hline & $\Delta_{\text {maks }}=$ & 0,018 & $<$ & $\Delta_{\mathrm{ijin}}=$ & 0,0562 & $\mathrm{~cm}$ & $\mathrm{OK}$ \\
\hline \multirow{3}{*}{ Girder GT 24} & $\sigma_{\text {maks }}=$ & 107,951 & $<$ & $\sigma_{\mathrm{ijin}}=$ & 70000 & $\mathrm{~kg} / \mathrm{cm}^{2}$ & $\mathrm{OK}$ \\
\hline & $\tau_{\text {maks }}=$ & 39,672 & $<$ & $\tau_{\mathrm{ijin}}=$ & 1400 & $\mathrm{~kg} / \mathrm{cm}^{2}$ & $\mathrm{OK}$ \\
\hline & $\Delta_{\text {maks }}=$ & 0,0001 & $<$ & $\Delta_{\mathrm{ijin}}=$ & 0,375 & $\mathrm{~cm}$ & $\mathrm{OK}$ \\
\hline \multirow{3}{*}{ Steel Wale SRZ } & $\sigma_{\text {maks }}=$ & 1023,163 & $<$ & $\sigma_{\mathrm{ijin}}=$ & 1200 & $\mathrm{~kg} / \mathrm{cm}^{2}$ & $\mathrm{OK}$ \\
\hline & $\tau_{\text {maks }}=$ & 236,607 & $<$ & $\tau_{\mathrm{ijin}}=$ & 696 & $\mathrm{~kg} / \mathrm{cm}^{2}$ & $\mathrm{OK}$ \\
\hline & $\Delta_{\text {maks }}=$ & 0,0001 & $<$ & $\Delta_{\mathrm{ijin}}=$ & 0,625 & $\mathrm{~cm}$ & $\mathrm{OK}$ \\
\hline Shoring & $\mathrm{P}_{\text {maks }}=$ & 2029,41 & $<$ & $P_{i j i n}=$ & 4000 & $\mathrm{~kg}$ & $\mathrm{OK}$ \\
\hline
\end{tabular}

(Source :Author, 2020) 
IV.3 Shoring Calculation for Beam 600*1200 mm

IV.3.1 Section Drawing

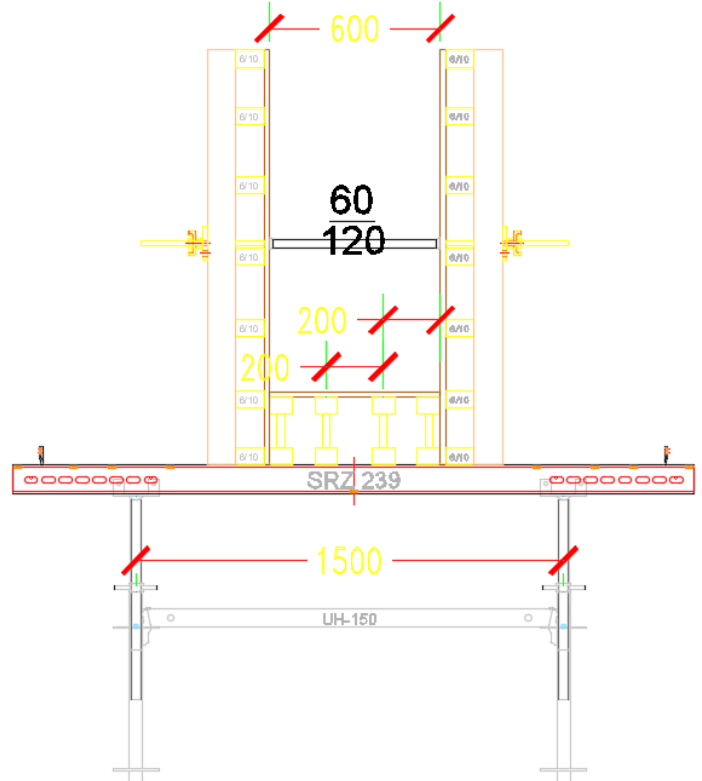

Figure 5. Upper Longitudinal Beam 600x1200 mm

(Source : Author, 2020)

\section{IV.3.2 Resume Control Stress, Strain, and Deflection}

The static calculation of the formwork shoring structure above, it can be concluded in Table 3 :

Table 3. Resume Control of Stress, Strain, and Deflection Shoring for Beam $60 * 120 \mathrm{~cm}$

\begin{tabular}{|c|c|c|c|c|c|c|c|}
\hline Item & \multicolumn{5}{|c|}{ Control Value } & Unit & Status \\
\hline \multirow{3}{*}{ Plywood 18 mm } & $\sigma_{\text {maks }}=$ & 16 & $<$ & $\sigma_{\mathrm{ijin}}=$ & 100 & $\mathrm{~kg} / \mathrm{cm}^{2}$ & $\mathrm{OK}$ \\
\hline & $\tau_{\text {maks }}=$ & 4,322 & $<$ & $\tau_{\text {ijin }}=$ & 12 & $\mathrm{~kg} / \mathrm{cm}^{2}$ & OK \\
\hline & $\Delta_{\text {maks }}=$ & 0,011 & $<$ & $\Delta_{\mathrm{ijin}}=$ & 0,0562 & $\mathrm{~cm}$ & $\mathrm{OK}$ \\
\hline \multirow{3}{*}{ Girder GT 24} & $\sigma_{\text {maks }}=$ & 932 & $<$ & $\sigma_{\mathrm{ijin}}=$ & 70000 & $\mathrm{~kg} / \mathrm{cm}^{2}$ & $\mathrm{OK}$ \\
\hline & $\tau_{\text {maks }}=$ & 33,552 & $<$ & $\tau_{\mathrm{ijin}}=$ & 1400 & $\mathrm{~kg} / \mathrm{cm}^{2}$ & $\mathrm{OK}$ \\
\hline & $\Delta_{\text {maks }}=$ & 0,021 & $<$ & $\Delta_{\mathrm{ijin}}=$ & 0,375 & $\mathrm{~cm}$ & OK \\
\hline \multirow{3}{*}{ Steel Wale SRZ } & $\sigma_{\text {maks }}=$ & 853 & $<$ & $\sigma_{\mathrm{ijin}}=$ & 1200 & $\mathrm{~kg} / \mathrm{cm}^{2}$ & OK \\
\hline & $\tau_{\text {maks }}=$ & 197,298 & $<$ & $\tau_{\mathrm{ijin}}=$ & 696 & $\mathrm{~kg} / \mathrm{cm}^{2}$ & $\mathrm{OK}$ \\
\hline & $\Delta_{\text {maks }}=$ & 0,001 & $<$ & $\Delta_{i j i n}=$ & 0,625 & $\mathrm{~cm}$ & $\mathrm{OK}$ \\
\hline Shoring & $\mathrm{P}_{\text {maks }}=$ & 1691,91 & $<$ & $\mathrm{P}_{\mathrm{ijin}}=$ & 4000 & $\mathrm{~kg}$ & $\mathrm{OK}$ \\
\hline
\end{tabular}

(Source :Author, 2020) 
IV.4 Calculation Shoring for Beam 800*1300 mm

IV.4.1 Section Drawing

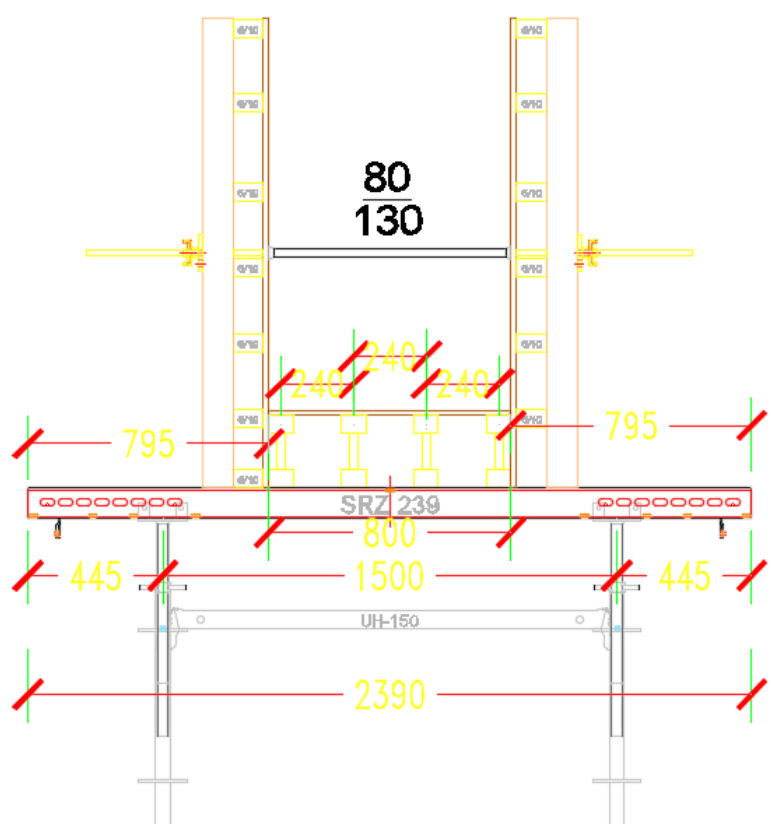

Figure 6. Upper Longitudinal Beam $800 \times 1300 \mathrm{~mm}$

(Source : Author, 2020)

\section{IV.4.2 Resume Control Stress, Strain, and Deflection}

The static calculation of the formwork shoring structure above, it can be concluded in Table 4. :

Table 4. Resume Control Stress, Strain, and Deflection Shoring for Casting Beam $80 * 130 \mathrm{~cm}$

\begin{tabular}{|c|c|c|c|c|c|c|c|}
\hline Item & \multicolumn{5}{|c|}{ Control Value } & Unit & Status \\
\hline \multirow{3}{*}{ Plywood 18 mm } & $\sigma_{\text {maks }}=$ & 31,58 & $<$ & $\sigma_{\mathrm{ijin}}=$ & 100 & $\mathrm{~kg} / \mathrm{cm}^{2}$ & $\mathrm{OK}$ \\
\hline & $\tau_{\text {maks }}=$ & 7,106 & $<$ & $\tau_{\mathrm{ijin}}=$ & 12 & $\mathrm{~kg} / \mathrm{cm}^{2}$ & $\mathrm{OK}$ \\
\hline & $\Delta_{\text {maks }}=$ & 0,023 & $<$ & $\Delta_{i j i n}=$ & 0,0562 & $\mathrm{~cm}$ & $\mathrm{OK}$ \\
\hline \multirow{3}{*}{ Girder GT 24} & $\sigma_{\text {maks }}=$ & 122 & $<$ & $\sigma_{\mathrm{ijin}}=$ & 70000 & $\mathrm{~kg} / \mathrm{cm}^{2}$ & $\mathrm{OK}$ \\
\hline & $\tau_{\text {maks }}=$ & 44,84 & $<$ & $\tau_{\mathrm{ijin}}=$ & 1400 & $\mathrm{~kg} / \mathrm{cm}^{2}$ & $\mathrm{OK}$ \\
\hline & $\Delta_{\text {maks }}=$ & 0,021 & $<$ & $\Delta_{\mathrm{ijin}}=$ & 0,375 & $\mathrm{~cm}$ & $\mathrm{OK}$ \\
\hline \multirow{3}{*}{ Steel Wale SRZ } & $\sigma_{\text {maks }}=$ & 1155,507 & $<$ & $\sigma_{\mathrm{ijin}}=$ & 1200 & $\mathrm{~kg} / \mathrm{cm}^{2}$ & OK \\
\hline & $\tau_{\text {maks }}=$ & 267,266 & $<$ & $\tau_{\mathrm{ijin}}=$ & 696 & $\mathrm{~kg} / \mathrm{cm}^{2}$ & $\mathrm{OK}$ \\
\hline & $\Delta_{\text {maks }}=$ & 0,001 & $<$ & $\Delta_{i j i n}=$ & 0,625 & $\mathrm{~cm}$ & $\mathrm{OK}$ \\
\hline Shoring & $\mathrm{P}_{\text {maks }}=$ & 2291,91 & $<$ & $P_{i j i n}=$ & 4000 & $\mathrm{~kg}$ & $\mathrm{OK}$ \\
\hline
\end{tabular}

(Source :Author, 2020)

\section{Conclusion}

Based on the analysis and recapitulation of the value of bending stress, deflection and shear stress in the beam formwork components of the Upper Longitudinal Beam:

a) Upper Longitudinal Beam formwork fulfills strength requirements with flexural stress values that occur on Plywood, Girder GT 24, and Steel Waller SRZ materials, each of which is smaller than $\sigma$ permit $=100 \mathrm{~kg} /$ $\mathrm{cm}^{2}, 70000 \mathrm{~kg} / \mathrm{cm}^{2}$, and $1200 \mathrm{~kg} / \mathrm{cm}^{2}$.

b) Upper Longitudinal Beam formwork meets the requirements of stiffness with deflection value occurring on Plywood and Girder GT 24 material is jin permit $=1 / 400$. Whereas the SRZ Steel Waller fulfills $\Delta$ permit $=1$ $/ 240$. 
VOLUME 2 | NUMBER 2 | MARCH 2020

Available online at http://proceedings.worldconference.id.

ISSN: 2656-1174 (online)

c) Upper Longitudinal Beam formwork fulfills the stability requirements with shear stress values that occur in Plywood, Girder GT 24, and SRZ Steel Waller materials, each of which is smaller than $\tau$ permit $=12 \mathrm{~kg} /$ $\mathrm{cm}^{2}, 1400 \mathrm{~kg} / \mathrm{cm}^{2}$, and $696 \mathrm{~kg} / \mathrm{cm}^{2}$.

d) Peri Up Scaffolding / Shoring is able to support the entire formwork of Upper Longitudinal Beam Size 90x100 cm, 60x120, and 80x130 with the respective values $\mathrm{P} \max =2029.41 \mathrm{~kg}, \mathrm{P} \max =1691.91 \mathrm{~kg}$, and $\mathrm{P}$ $\max =2291.91 \mathrm{~kg}$. Where the 1 foot shoring capacity is able to withstand a permit load of $\mathrm{P}=4000 \mathrm{~kg}$.

\section{References}

[1] Dewobroto W. \& Marianto H. (2015): “Analisis Stabilitas Perancah Bertingkat dengan Advance Analysis dan D.A.M.", Prosiding Seminar Nasional: Sains, Rekayasa \& Teknologi UPH-2015, Karawaci, Tangerang, Google Scholar.

[2] Dipohusodo, I. (1988): “Mengenal Acuan Beton Bertulang”, Yogyakarta: Liberty.

[3] Doloksaribu B. (2018): “Analisa Perhitungan Kekuatan Perancah Terhadap Waktu Siklus Pengecoran Lantai untuk Memenuhi Keamanan Struktur Bangunan”. Universitas Medan Area. Google Scholar.

[4] F. Wigbout Ing. (1997): “ Bekisting (Kotak Cetak) “, Jakarta: Erlangga.

[5] Fitriansyah, K. R. (2018): “Analisis Pada Pelaksanaan Pekerjaan Bekisting Plat Lantai Menggunakan Bekisting Ring-Lock Scafolding Dan Aluma System Terhadap Perbandingan Biaya Dan Waktu (Studi Kasus: Kota Kasablanka 3)”, Universitas Mercu Buana Jakarta. Google Scholar.

[6] Hidayat A. ST., MT. (2018): “Buku Panduan Penulisan Proposal \& Tugas Akhir”, Fakultas Teknik Sipil, Universitas Mercu Buana.

[7] Husniah, F. (2017): "Metode Pelaksanaan, Analisis Kekuatan dan RAB Bekisting Semi Sistem Struktur Sub Dam 1 WO-C2 Rehabilitasi dan Rekonstruksi Sabo Dam Merapi Kali Woro Prov. Jawa Tengah”, Universitas Gadjah Mada. Google Scholar.

[8] Ir. V Sunggono Kh. (1984): “ Buku Teknik Sipil “, Bandung: Nova.

[9] NI-2 (1971). Peraturan Beton Bertulang Indonesia, Indonesia

[10] NI-3 (1970). Peraturan Umum untuk Bahan Bangunan di Indonesia.

[11] NI-5 (1961). Peraturan Konstruksi Kayu Indonesia.

[12] Nugraha, H. R. (2018): “Evaluasi Terhadap Penggunaan Perancah Baja Modifikasi Sebagai Penopang Sementara Untuk Struktur Transfer Beam Column”, Universitas Jember, Google Scholar.

[13] Pamungkas C.C. (2016): ’Design Pemodelan Bekisting System Untuk Pelat 60 Cm (Studi Kasus : Proyek Gedung BRI PSCF Ragunan)”, Universitas Pancasila.

[14] Peraturan Standard Beton 1991 (SK.SNI T-15-1991-03).

[15] Saptowati, H. (2018): “Analisis Bekisting Pada Pengecoran Dinding Bunker Gedung Iradiator MerahPutih”. PRIMA-Aplikasi dan Rekayasa dalam Bidang Iptek Nuklir, 15(2), 1-9.

[16] Sudarmono; Setiono K. J.; Anung Suwarno A. (2015): ”Sistem Acuan Perancah Balok Lantai Yang Mudah Dipasang Bongkar Tanpa Tiang”, Politeknik Negeri Semarang 
[17] Talim, M. (2017): “Analisis Pengaruh Kuat Tekuk pada Sistem Perancah Bangunan (Scaffolding) dengan Metode Analisa Langsung (Direct Analysis Method)”, Universitas SumateraUtara, Google Scholar.

[18] Zakkiyah, N. (2015): “Analisis Kekuatan, Kekakuan dan Stabilitas Bekisting Pada Proyek Pembangunan UI Teaching Hospital”, Universitas Gadjah Mada, Google Scholar.

\section{Biographies}

Agyanata Tua Munthe was born in 21 March 1981. After graduation his highschool education at BPK Penabur senior high school in Bandar Lampung, continued his civil engineering education at Atmajaya University, Special Region of Yogyakarta in 2004. He obtained his Master's degree in civil engineering in 2006 at University of Gajah Mada, Special of Region of Yogyakarta. Currently active as lecturer at Mercu Buana University Jakarta with an interest in structure part and active as a project manager managing several construction projects.

Muhammad Ardiansyah Noegroho was born in 19 March 1990 at Palopo City, South of Sulawesi in Indonesia. He completed his study at Politeknik Negeri Ujung Pandang for his Associate/Diploma Degree in 2011. He continued his study at Universitas Mercu Buana in 2018-2020 for his bachelor degree. Currently, he worked at PT. Adhi Karya as Project QHSE Manager for Light Rail Transit (LRT) Jabodebek Project. 